Check for updates

Cite this: RSC Adv., 2018, 8, 20263

\title{
Tapping into cyanobacteria electron transfer for higher exoelectrogenic activity by imposing iron limited growth $\uparrow$
}

\begin{abstract}
A. C. Gonzalez-Aravena, ${ }^{a}$ K. Yunus, ${ }^{a}$ L. Zhang, ${ }^{\text {b }}$ B. Norling ${ }^{\mathrm{b}}$ and A. C. Fisher (DD *a
The exoelectrogenic capacity of the cyanobacterium Synechococcus elongatus PCC7942 was studied in iron limited growth in order to establish conditions favouring extracellular electron transfer in cyanobacteria for photo-bioelectricity generation. Investigation into extracellular reduction of ferricyanide by Synechococcus elongatus PCC7942 demonstrated enhanced capability for the iron limited conditions in comparison to the iron sufficient conditions. Furtheremore, the significance of $\mathrm{pH}$ showed that higher rates of ferricyanide reduction occurred at $\mathrm{pH}$ 7, with a 2.7-fold increase with respect to $\mathrm{pH} 9.5$ for iron sufficient cultures and 24 -fold increase for iron limited cultures. The strategy presented induced exoelectrogenesis driven mainly by photosynthesis and an estimated redirection of the $28 \%$ of electrons from photosynthetic activity was achieved by the iron limited conditions. In addition, ferricyanide reduction in the dark by iron limited cultures also presented a significant improvement, with a 6 -fold increase in comparison to iron sufficient cultures. Synechococcus elongatus PCC7942 ferricyanide reduction rates are unprecedented for cyanobacteria and they are comparable to those of microalgae. The redox activity of biofilms directly on ITO-coated glass, in the absence of any artificial mediator, was also enhanced under the iron limited conditions, implying that iron limitation increased exoelectrogenesis at the outer membrane level. Cyclic voltammetry of Synechococcus elongatus PCC7942 biofilms on ITO-coated glass showed a midpoint potential around $0.22 \mathrm{~V}$ vs. Ag/ $\mathrm{AgCl}$ and iron limited biofilms had the capability to sustain currents in a saturated-like fashion. The present work proposes an iron related exoelectrogenic capacity of Synechococcus elongatus PCC7942 and sets a starting point for the study of this strain in order to improve photo-bioelectricity and darkbioelectricity generation by cyanobacteria, including more sustainable mediatorless systems.
\end{abstract}

Received 31st January 2018

Accepted 14th May 2018

DOI: 10.1039/c8ra00951a

rsc.li/rsc-advances resulting in an intracellular electron transport chain. Bioelectricity from thylakoid membrane isolated from the cell apparatus has been achieved in response to light. ${ }^{4-6}$ However, whole microbes have the advantage of being a self-sustained and self-repairing, which greatly impacts the durability of the bioelectrochemical cell.

Electricity generated by photosynthetic microorganisms has been demonstrated in a range of cyanobacteria and microalgae species utilised as electron donor in biophotovoltaics (BPV, photosynthesis driven bioelectrochemical cell). Studies have shown a direct correlation with electricity and increased illumination, furthermore electricity generated in the absent of light is also generated as the result of dark metabolic activity. ${ }^{7-9}$ In biotechnological applications, including bioelectricity, special attention has been given to prokaryotic cyanobacterial species due to their simpler physiology and lower energy requirements. ${ }^{\mathbf{1 0 , 1 1}}$

Bombelli et $a l .{ }^{4}$ used various photosynthesis inhibitors to demonstrate that the intracellular electron donor in the exoelectrogenic path is $\mathrm{NAD}(\mathrm{P}) \mathrm{H}$. However, the exoelectrogenic capacity in cyanobacteria is not constitutive and electrons
${ }^{a}$ Department of Chemical Engineering and Biotechnology, University of Cambridge, Philippa Fawcett Drive, Cambridge, CB3 OAS, UK. E-mail: acf42@cam.ac.uk ${ }^{b}$ School of Biological Sciences, Nanyang Technological University, 637551, Singapore $\dagger$ Electronic supplementary information (ESI) available. See DOI: 10.1039/c8ra00951a 
generated in the photolysis of water are directed to $\mathrm{CO}_{2}$ fixation, it is only a very small fraction reaching out the cell surface. ${ }^{12}$ The mechanisms and metabolic pathways involved in the redirection of electrons to the extracellular membrane are a matter of study and yet to be fully understood. ${ }^{\mathbf{1 3 , 1 4}}$

Good exoelectrogenic bacteria are those coupling respiration to an extracellular final electron acceptor, and examples of exoelectrogens such as Shewanella oneidensis and Geobacter sulfurreducens have been reported extensively in the literature. In both cases, outer membrane c-cytochromes associated to extracellular respiratory pathways are responsible of direct electron transfer. ${ }^{15-17}$ It has also been reported the secretion of redox active compounds like riboflavins, which can act as a natural redox mediator. ${ }^{18,19}$ Additionally, the presence of electrically conductive nanowires (pili) on the outer surface of the bacterial wall has been described as necessary for higher extracellular electron transport rates..$^{20-22}$ Cyclic voltammetry of these bacterial biofilms shows electrochemical active regions. $^{23,24}$

In the case of poor exoelectrogens like photosynthetic microorganisms, soluble artificial redox mediators impermeable to the plasma membrane, typically ferricyanide, are utilised to facilitate exoelectrogenesis (Fig. 1). ${ }^{\mathbf{1 2 , 2 5}}$ Nevertheless, biofilms of photosynthetic microorganisms are capable of electricity generation with no addition of mediators (mediatorless), revealing direct electron transfer, ${ }^{7}$ and potentially representing a more sustainable platform.

While for cyanobacteria there is a lack of understanding on how exoelectrogenesis occurs, eukaryotic microalgae present proteins with a conserved transmembrane ferric reductase domain (FRD superfamily) including ferric reductases (FRE) and NADPH oxidases (NOX). ${ }^{26}$ FRE and NOX perform vertical electron transfer through the plasma membrane, therefore constituting a form of exoelectrogenesis. FRE activities can be analysed using the ferricyanide assay, ${ }^{27}$ which consists of measuring the electrochemical conversion of ferricyanide $\left(\mathrm{Fe}(\mathrm{CN})_{6}{ }^{-3}\right)$ to ferrocyanide $\left(\mathrm{Fe}(\mathrm{CN})_{6}{ }^{4-}\right)$, giving a quantitative

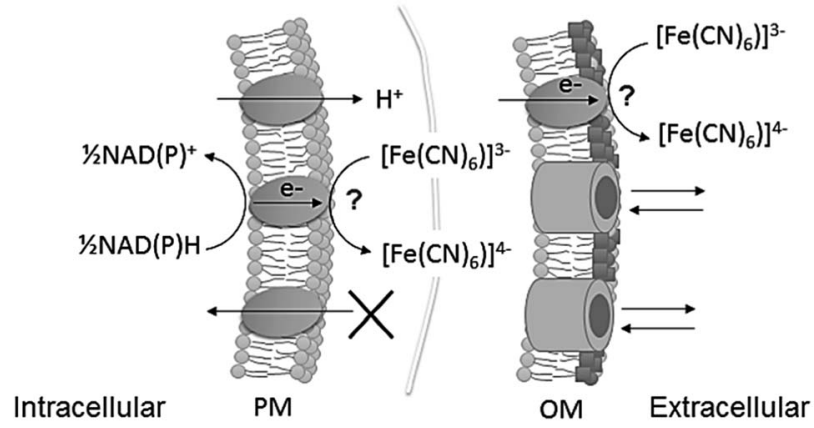

Fig. 1 Schematic of ferricyanide reacting at the extracellular surface of cyanobacteria, at plasma membrane (PM) and at outer membrane (OM) level. The points of reaction have not been identified. Ferricyanide/ferrocyanide can cross the OM through porins, but not the PM. $\mathrm{NADPH}$ and $\mathrm{NADH}$ are the intracellular metabolites known to be the substrate for the reaction. ${ }^{13}$ Ferrocyanide is then available in the extracellular space to react with an electrode or alternatively, the reaction can be used to quantify exoelectrogenesis by measuring ferricyanide concentration spectrophotometrically. measurement of their exoelectrogenesis. FRE and NOX in the plasma membrane could explain the exoelectrogenic capacity observed in microalgae based BPVs. ${ }^{9,28}$ Furthermore, the algal plasma membrane NADPH oxidase was identified to be responsible for extracellular superoxide production which was correlated with current generation in BPV studies. ${ }^{25,29}$

In the case of cyanobacteria, some authors ${ }^{10,30}$ have suggested that exoelectrogenesis may be due to a reductive iron uptake mechanism as well. Although, proteins with the conserved ferric reductase domain have not been annotated for cyanobacteria. ${ }^{26}$ The strategy for iron uptake described for cyanobacteria consists of siderophore secretion for ferric chelation, followed by the uptake of the $\mathrm{Fe}(\mathrm{III})$-siderophore complex. ${ }^{31-33}$ However, many cyanobacteria do not produce siderophores, therefore reductive mechanisms are being sought and evidence of possible reductive iron uptake pathways is growing. ${ }^{34-37}$ New insight on cyanobacterial iron acquisition has revealed ferric reduction due to the action of the Alternate Respiratory Terminal Oxidase (ARTO) in the plasma membrane with the subsequent uptake of ferrous, particularly described for the model cyanobacterium Synechocystis sp. PCC6803, ${ }^{34,38,39}$ a common strain used in biophotovoltaics. However, Bradley et $a .^{\mathbf{1 2}}$ demonstrated that deletion of ARTO increases ferricyanide reduction rates in Synechocystis sp. PCC6803, thus a different point of electron export must exist.

The model cyanobacterium Synechococcus elongatus PCC7942 (PCC7942 hereafter), has been broadly studied in iron starvation, ${ }^{\mathbf{4 0}-43}$ having particularly high endurance to iron limited growth. ${ }^{\mathbf{4 4}}$ PCC7942 is a siderophore producer, with increased siderophores secretion and overexpression of ferric transporters under iron starvation..$^{31,45-47}$ However, Nodop et al. $^{47}$ suggested multiple iron acquisition systems. In their work, the transcript profile of iron starved PCC7942 showed upregulation of proteins in the cell envelope, including a porin in the outer membrane (SomB1), the operon IrpAB, involving a plasma membrane protein (IrpA) and a c-type cytochrome (IrpB), and a high affinity Fe(II) permease (ftr1), all regulated by the same transcriptional factor. ${ }^{47}$ This system indicates that possibly a reductive mechanism under iron starvation is activated.

Recently, PCC7942 has been reported as an exoelectrogen for photo-bioelectricity generation. ${ }^{48}$ Moreover, PCC7942 was transformed with the outer membrane c-type cytochrome $\mathrm{S}$ (OmcS) from Geobacter sulfurreducens, improving PCC7942 exoelectrogenic capacity with increased ferricyanide reduction rates as well as higher mediatorless electricity generation. In order to develop platforms for direct current generation from cyanobacteria it is imperative to understand their exoelectrogenic mechanisms and to find conditions favouring higher rates of exoelectrogenesis. In the present study we report our findings on PCC7942 exoelectrogenesis, based on measurements of extracellular ferricyanide reduction rates, demonstrating that by imposing iron limited growth, exceptionally higher exoelectrogenic capacity is achieved. Furthermore, voltammetry measurements were performed in absence of an artificial mediator, showing that direct electricity generation increases for iron limited PCC7942. 


\section{Experimental}

\section{Microorganisms culturing conditions}

PCC7942 was obtained from the Pasteur Culture Collection. PCC7942 was grown in BG11 medium. Growth was at $30{ }^{\circ} \mathrm{C}$ under a 12 hours light/dark cycle illuminated with white light fluorescent tubes with an intensity of $90 \mu \mathrm{mol} \mathrm{m} \mathrm{m}^{-2} \mathrm{~s}^{-1}$ and shaking speed of $120 \mathrm{rpm}$ in air.

Erlenmeyer flasks with vented caps $(0.22 \mu \mathrm{m}$ PTFE) were used. Cultures were routinely tested to check axenic conditions by plating in bacteriological agar (tryptone soya with yeast extract); plates were wrapped in aluminium foil and incubated at $30{ }^{\circ} \mathrm{C}$ for four days to check absence of bacterial growth.

\section{Iron limitation culturing}

Media for experiments was prepared with Millipore ${ }^{\circledR}$ ultrapure water (polished R.O. water $18.2 \mathrm{M} \Omega \mathrm{cm}$ at $25^{\circ} \mathrm{C}$ ). For the iron limited condition, ammonium ferric citrate in BG11 was replaced by ammonium citrate in a stoichiometric proportion. Iron presence in other chemical reagents meant that traces of iron were still introduced.

All glassware was acid-washed to remove solids and thus minimize iron presence. Bottles and flasks were left overnight with $1 \mathrm{M}$ nitric acid and then rinsed with ultrapure water.

Triplicates of iron sufficient and iron limited cultures were started from a common culture, which was divided into two, centrifuged at $4,000 \times g$ for 10 minutes, with two washing steps using fresh media. Cultures of $200 \mathrm{ml}$ were prepared with an initial optical density $\left(\mathrm{OD}_{750 \mathrm{~nm}}\right)$ of around 0.5 corresponding to biomass concentration of $0.13 \mathrm{mg} \mathrm{ml}^{-1}$ for PCC7942 and left to grow for 14 days. Spectrophotometric readings were obtained using a Thermo Scientific Evolution 201 UV-Visible spectrophotometer.

For chlorophyll content, $1 \mathrm{ml}$ of culture was centrifuged at high speed for $1 \mathrm{~min}, 0.9 \mathrm{ml}$ of supernatant was removed and $0.9 \mathrm{ml}$ of $99.9 \%$ methanol (Sigma Aldrich) was added, samples were left for at least $15 \mathrm{~min}$ in the dark before centrifuging again, absorbance was measured at $665 \mathrm{~nm}$; an extinction coefficient of $12.9447 \mu \mathrm{g} \mathrm{ml}^{-1}$ was used for calculations. ${ }^{49}$

Photosynthesis rate was estimated by measuring oxygen evolution in light and darkness with a Clark electrode (Hanna Instrument ${ }^{\circ}$ DO meter HI-2040 edge () . Gross photosynthesis was calculated as the addition of net photosynthesis and respiration.

\section{Ferricyanide assay}

Samples of $6 \mathrm{ml}$ with a biomass concentration of $0.53 \mathrm{mg} \mathrm{ml}$ $\left(\mathrm{OD}_{750 \mathrm{~nm}}\right.$ of 2$)$ were obtained by centrifugation $(4,000 \times \mathrm{g}$ for 10 min). Pellets were re-suspended in a modified BG11 media lacking ammonium iron citrate, citric acid and EDTA (BG11(-) $\times 3$ ) in order to avoid redox active compounds. For measurements in the dark, cultures were placed in vented flasks, wrapped in aluminium foil and incubated for at least 3 hours before centrifugation and resuspension. The $6 \mathrm{ml}$ samples were left to acclimatise for at least one hour before proceeding to measure exoelectrogenic activity. Potassium ferricyanide (Sigma
Aldrich) was added to each sample from a $100 \mathrm{mM}$ stock solution. Aliquots of $1 \mathrm{ml}$ were filtered at 0,10, 30 and 120 minutes. The filtrate was analysed for ferricyanide spectrophotometrically at $420 \mathrm{~nm}$, (using an extinction coefficient of 1.052 $\mathrm{mM} \mathrm{cm}^{-1}$ ). Ferricyanide reduction rates were calculated by linear regression.

Photosynthesis inhibition was imposed by adding DCMU (3(3,4-dichlorophenyl)-1,1-dimethylurea, Sigma Aldrich) to BG11 $(-) \times 3$. Stock solution of DCMU (9 mM) was prepared on 95\% ethanol (Alfa Aesar). A volume of $11.7 \mu$ l of DCMU solution was added to $7 \mathrm{ml}$ of $0.53 \mathrm{mg} \mathrm{ml} \mathrm{m}^{-1}$ samples, for a final concentration of $15 \mu \mathrm{M}, 50$ minutes before adding potassium ferricyanide and analysed as described. $11.7 \mu \mathrm{M}$ of $95 \%$ ethanol was added to non-inhibited samples and no effect was found.

When necessary, $\mathrm{BG} 11(-) \times 3$ was buffered to maintain specific $\mathrm{pH}$. HEPES-NaOH was added at a concentration of $10 \mathrm{mM}$ to maintain a neutral $\mathrm{pH}(\mathrm{pH}=7.0)$, and bicarbonate at $0.1 \mathrm{M}$ was added to maintain alkaline $\mathrm{pH}(\mathrm{pH}=9.5)$. Blanks of ferricyanide conversion in $\mathrm{BG} 11(-) \times 3$ were also conducted showing no significant reduction.

\section{pH evolution}

$\mathrm{pH}$ evolution in iron limited and iron sufficient samples $(30 \mathrm{ml})$, and upon addition of $1 \mathrm{mM}$ ferricyanide, was continuously monitored and recorded every 30 seconds using a Picolog® DrDAQ data logger and DD011 pH electrode.

\section{Working electrode fabrication and operation}

The electrochemical platform consisted of a working electrode (WE) fixed at the bottom of a cylindrical chamber of $50 \mathrm{~mm}$ internal diameter and with a well of $18 \mathrm{~mm}$ diameter (Fig. 2). The WE had a $18 \mathrm{~mm}$ diameter active electrode area. A titanium strip was placed as electrical contact for the WE. The counter electrode (CE) was 99.9\% platinum mesh (Alfa Aesar) and an Ag/ $\mathrm{AgCl}$ reference electrode (RE, MF-2052 RE-5B Basi®) was used. The latter were introduced in the chamber just on the day of measurements. The chamber assembled with the WE was sterilised by autoclaving. To keep sterile conditions, good ventilation and illumination, the cylinder top was covered with a piece of transparent PET and a vented area using nitrocellulose paper $(0.2 \mu \mathrm{m}$ pore size), which was sealed with antibacterial tape.

The well of the device was inoculated with cultures of iron limited or iron sufficient cultures (10 days old) and left to settle down. After four days electrochemical measurements (cyclic voltammetry) were conducted in an incubator at $30 \pm 1{ }^{\circ} \mathrm{C}$ under aerated conditions. To study the biofilms, on the day of measurements the supernatant was carefully removed and replaced with fresh modified media BG11 $(-) \times 3$. To obtain thinner biofilms, these were carefully washed repeated times to remove the more loosely attached cells. For measurements under illumination, light intensity was $90 \mu \mathrm{mol} \mathrm{s} \mathrm{s}^{-1} \mathrm{~m}^{-2}$. Measurements in the dark were carried out after 3-4 hours of dark adaptation. Electrochemical measurements were conducted using a PalmSens MultiEmStat ${ }^{3}$ four-channel potentiostat, integrated with the MultiTrace software. 


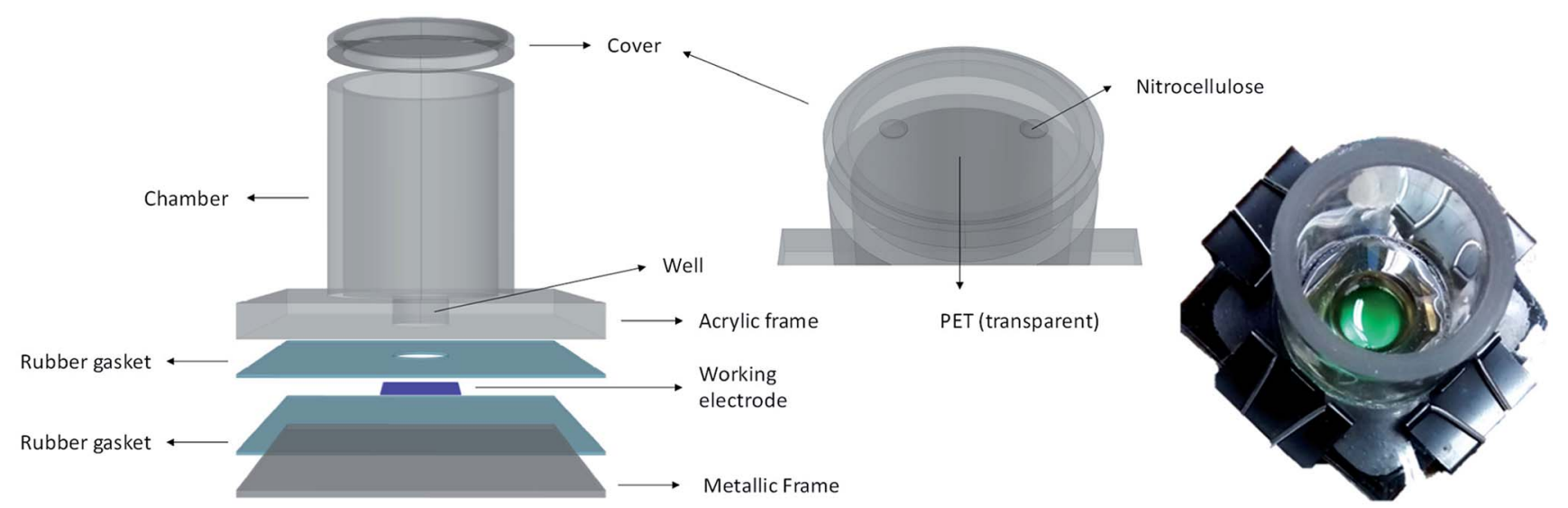

Fig. 2 Schematics and photography of device holding the working electrode.

\section{Biofilm cryo-immobilisation and SEM imaging}

To observe biofilm formation on ITO-coated glass, PCC7942 was inoculated in a transparent tube of $10 \mathrm{~mm}$ diameter with a piece of ITO-coated glass at the bottom and left to settle down. The piece was collected and submerged in cold water to carefully wash off non-attached cells. To observe thinner biofilms, additional washing steps were carried out. Biofilms were quench frozen by plunging, cell face first into melting ethane cooled in liquid nitrogen. They were freeze dried in a Quorum/ Emitech K775X. The dried biofilms were coated with $16 \mathrm{~nm}$ of iridium in a Quorum/Emitech K575X sputter coater. Biofilms were viewed in a FEI Verios 460L operated at $2 \mathrm{kV}$ and $50 \mathrm{pA}$.

\section{Results and discussion}

\section{PCC7942 exoelectrogenesis under iron limited growth}

In order to investigate extracellular electron transfer in PCC7942 for applications in photo-bioelectricity generation, the relationship between iron limited growth and exoelectrogenesis was evaluated. Iron limited and iron sufficient cultures of PCC7942 were studied in their capacity to reduce ferricyanide (FeCN-R rates), commonly used in ferric reductase assays and as an artificial mediator in biophotovoltaics platforms. ${ }^{4,27,50}$ The effect of ferricyanide/ferrocyanide on the metabolism of cyanobacteria has been described as non-toxic at low concentrations (below 50 $\mathrm{mM}$ ), as this iron complex is impermeable to the plasma membrane. ${ }^{4}$ In this study the ferricyanide assay was conducted for only 2 hours, thus eliminating any possible long term effect.

Culturing conditions were similar to those encountered in BPV experiments, which typically consist of a light/dark cycle with temperatures up to $30^{\circ} \mathrm{C}$, alkaline $\mathrm{pH}$ and in aerated conditions. It has been reported that growth rates of PCC7942 do not increase above the 100-120 $\mu \mathrm{mol} \mathrm{m} \mathrm{m}^{-2} \mathrm{~s}^{-1,51,52}$ therefore experiments were conducted under a light intensity of $90 \mu \mathrm{mol} \mathrm{m} \mathrm{m}^{-2} \mathrm{~s}^{-1}$. However, high growth rates are normally obtained under continuous illumination and supplying air enriched in $\mathrm{CO}_{2}(1-5 \%) .{ }^{52}$ Therefore, experimental conditions resulted in relative low growth rates with doubling times of around 5 days (ESI Fig. $1 \dagger$ ) consequently, iron requirements (consumption) were lessened and it was observed that PCC7942 growing in iron limited media maintained the same growth rate as cells grown in iron sufficient conditions in the time frame studied (14 days). Likewise, chlorophyll content was maintained in the same levels and photosynthetic and respiration rates were not significantly different (ESI Fig. $1 \dagger$ ). Concomitantly, iron limited cultures increased their exoelectrogenic capacity in time (Fig. 3). The reduction rates of iron limited cultures in light (90 $\left.\mu \mathrm{mol} \mathrm{m}^{-2} \mathrm{~s}^{-1}\right)$ increased from around $45 \operatorname{pmol}_{\left(\left[\mathrm{Fe}(\mathrm{CN})_{6}\right]^{3-}\right)}$ $\mathrm{nmol}_{\mathrm{Chl}}{ }^{-1} \min ^{-1}$ (Day 2) to $460 \mathrm{pmol}_{\left(\left[\mathrm{Fe}(\mathrm{CN})_{6}\right]^{3-}\right)} \mathrm{nmol}_{\mathrm{Chl}}^{-1} \mathrm{~min}^{-1}$
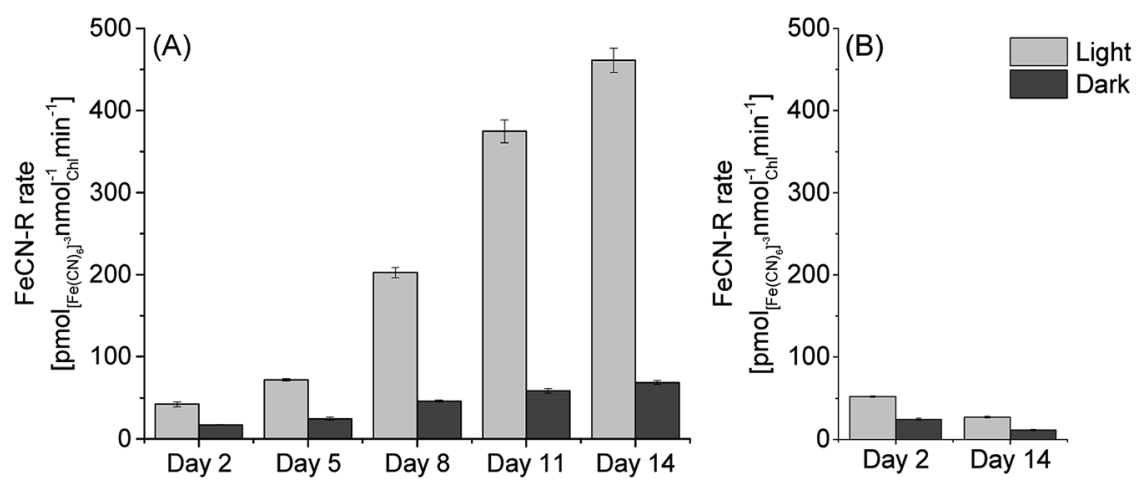

Fig. 3 Ferricyanide reduction rates of PCC7942 (A) in iron limited conditions and (B) in iron sufficient conditions, for different days of culture growth (assayed at $\mathrm{pH}$ 7.0). FeCN-R rate $n=3 \pm 1 \mathrm{SE}$. 
(Day 14), a 10-fold increase and equivalent to a 17 -fold increase compared with the rates of iron sufficient cultures (Day 14).

In the dark, FeCN-R rates were considerably lower than those in the light. However, it is noticeable that iron limited cultures also experienced an increase in dark exoelectrogenesis. After 14 days, iron limited cells developed FeCN-R rates in the dark six times higher than those of iron sufficient cells. Increased rates in the dark represent an important improvement for the development of BPV platforms which will be operated in daylight/night cycles.

Rates were measured at $\mathrm{pH} 7.0$ because they were found to be higher around neutral pH (Fig. 4), even though alkaline $\mathrm{pH}$ (9.5) is typical of cyanobacteria cultures. Considering the effect of $\mathrm{pH}$ alone (iron sufficiency), a 2.7-fold increase in FeCN-R rates was obtained, while a 24-fold increase is the total effect on iron limited PCC7942 if compared to rates from iron sufficient cultures at alkaline $\mathrm{pH}$.

Proteins with a ferric reductase motif have not been described for cyanobacteria ${ }^{26}$ To further confirm that PCC7942 does not encode ferric reductases, its genome was queried for conserve domains using the NCBI web-based tool Batch CD-Search. ${ }^{53} \mathrm{~A}$ list of conserved domains for all PCC7942 genes was obtained and no proteins with the ferric reductase annotation are present.

Interestingly, the $\mathrm{pH}$-dependence of the FeCN-R activity detected in PCC7942 is like that of ferric reductases in plants and algae, with optimal $\mathrm{pH}$ of ferric reductases in the plasma membrane as low as pH 5.5 (ref. 54) and inhibited at high $\mathrm{pH}^{54-58}$ Regulated paths which operate optimally at a more acidic $\mathrm{pH}$ as a strategy for iron uptake is likely due to the fact that lower $\mathrm{pH}$ favours the solubility of ferrous, the product of ferric reduction. ${ }^{59}$ The enhanced ferricyanide reduction capacity of iron limited PCC7942 may be indirect evidence of the proposed reductive iron acquisition system involving SomB1-irpAB-ftr1, overexpressed in iron limited growth. ${ }^{47}$

As reported for ferric/ferricyanide reductases in microalgae and plants, ${ }^{\mathbf{5 4 5 6 , 6 0 , 6 1}}$ PCC7942 caused extracellular acidification in unbuffered media, as a consequence of extracellular electron transport to ferricyanide (Fig. 5). Acidification occurs due to the polarisation of the plasma membrane and charge compensation, although other cations different to protons could be extruded instead. ${ }^{60}$ Fig. 5 shows that by adding ferricyanide in the light phase (some noise was introduced due to disturbing the probe), $\mathrm{pH}$ drops at a minimal pace. However, after the dark

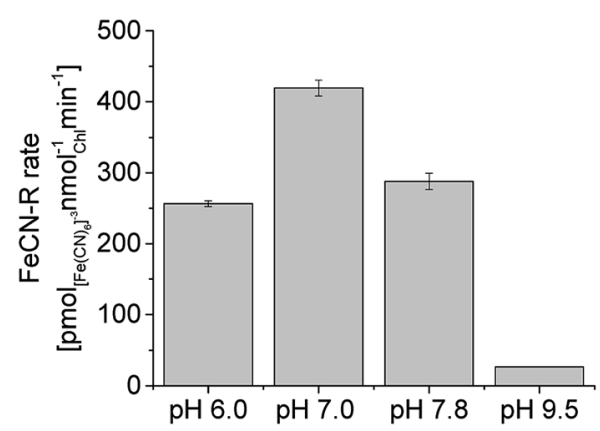

Fig. 4 Ferricyanide reductase activity of iron limited PCC7942 cultures (14 days old) at different $\mathrm{pH}$, under illumination. FeCN-R rate $n=3 \pm$ $1 \mathrm{SE}$. phase, when the $\mathrm{pH}$ dropped due to absence of photosynthetic activity, and in the subsequent illumination stage, the conditions of $\mathrm{pH}$ and photosynthetic activity co-existed for high FeCN-R rates to proceed, which in turn caused a steep drop in $\mathrm{pH}$ for the iron limited condition. The latter makes buffers necessary to keep good culturing conditions, although in working BPV platforms, proton consumption by the air cathode will compensate acidification.

The light-dependence of electron transfer to ferricyanide from PCC7942 is clear, both in the ferricyanide conversion measurements and in the $\mathrm{pH}$ evolution measurements. The intracellular substrate for extracellular electron transfer in cyanobacteria has been reported to be $\operatorname{NAD}(\mathrm{P}) \mathrm{H},{ }^{4}$ which increases under illumination due to the contribution of photosynthesis, and in turn provides a source of electrons (reducing power) and protons. To confirm the participation of photosynthetic NADPH in the exoelectrogenic activity of iron limited PCC7942, the ferricyanide assay was conducted in three different conditions: light $\left(90 \mu \mathrm{mol} \mathrm{m} \mathrm{m}^{-2} \mathrm{~s}^{-1}\right)$, light with addition of DCMU (photosynthesis inhibitor) and darkness (Fig. 6). In the light, after addition of DCMU, FeCN-R rates were strongly inhibited, confirming that PCC7942 exoelectrogenesis is mainly driven by the reducing power generated in photosynthesis. The respiratory pathway and the biosynthesis oxidative pentose phosphate pathway are other sources of intracellular reducing power, with generation of NADH and NADPH respectively, which can drive dark exoelectrogenesis. Iron limitation also induces higher rates independent of photosynthesis (dark and DCMU inhibited), indicating that the NAD(P)H pool from these two pathways are also available under iron limited growth.

As observed, the high FeCN-R rates obtained for iron limited PCC7942 under illumination are strongly dependent on photosynthesis, implying that extracellular electron transfer is actively competing with $\mathrm{CO}_{2}$ fixation. The low exoelectrogenic activity of photosynthetic microorganisms has been discussed and $\mathrm{CO}_{2}$ fixation accounted as the main competitor for electrons. ${ }^{12,13}$ Our results show that by exposing PCC7942 cultures to iron limited growth, exoelectrogenesis can favourably compete for reducing power, suggesting that there is an active mechanism redirecting electrons to the cell surface, possibly involving electron transfer by IrpB (oxidoreductase), which is overexpressed under iron starvation together with IrpA (plasma membrane protein), ftr1 (ferrous uptake) and SomB1 (outer membrane porin), all being part of a strategy to increase iron uptake capacity. ${ }^{47}$

Reported value of electrons from photosynthesis redirected extracellularly to ferricyanide is approximately $0.3 \%$ in the cyanobacterium Synechocysistis sp. PCC6803 (wild-type). ${ }^{12}$ We estimate a redirection of reducing power from photosynthesis in the order of $1 \%$ by iron sufficient PCC7942 at neutral $\mathrm{pH}$ and $28 \%$ by iron limited PCC7942 at neutral pH (ESI eqn (1) $\dagger$ ), considering photosynthetic oxygen production of $350 \mathrm{pmol}$ $\mathrm{nmol}_{\mathrm{chl}}{ }^{-1} \min ^{-1}$, determined for PCC7942 in the studied conditions (ESI Fig. $1 \dagger$ ).

Light intensity and wavelength are other factors affecting oxygen evolution in the photosynthetic process, thus generation of reducing power. For instance, McCormick at el. ${ }^{7}$ showed that 

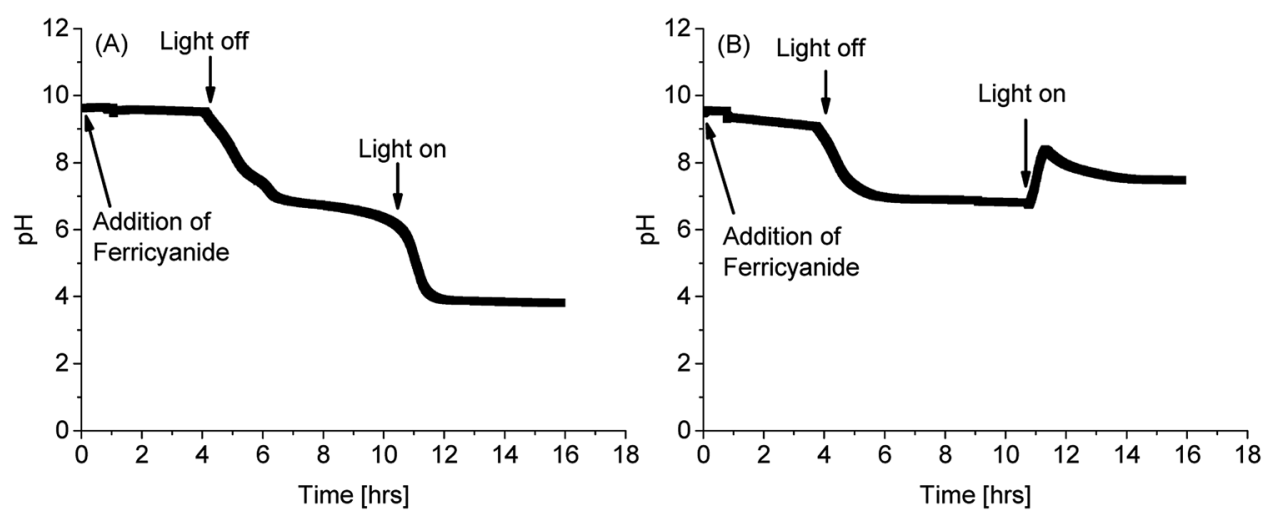

Fig. $5 \mathrm{pH}$ evolution of PCC7942 cultures (Day 14) in unbuffered media with addition of $1 \mathrm{mM}$ of ferricyanide (A) in iron limitation and (B) in iron sufficiency.

red light induced the highest photosynthetic rates and the highest power in a mediatorless BPV when using the cyanobacterium Synechococcus sp. WH5701. In a ferricyanide mediated BPV platform studied by Bombelli et al., ${ }^{4}$ light intensity did not show a great influence, as it was seen that the maximum power output was independent of light intensity, although the authors also showed that the time to reach peak power was longer at low light intensities. Therefore, at higher light intensities photosynthesis allowed a more rapid generation of intracellular reducing power, but ultimately the "bottleneck" is at electron export level. The effect of light intensity over the exoelectrogenic capacity of iron limited PCC7942 is to be studied, while higher light intensity will increase reducing power generation, iron limitation causes stressing conditions for photosynthesis, because there is a minimal requirement of $22-23$ iron atoms for a complete functional photosynthetic apparatus. ${ }^{62}$ Therefore, a fine balance between light intensity, iron limitation and extracellular electron capture will be necessary.

Additionally, the effect of iron limitation over other metabolic processes should be studied. Recently, PCC7942 has been described as a potential candidate for biodiesel feedstock thanks to its lipid composition, primary fatty acids are $\mathrm{C} 16-\mathrm{C} 18$, which is suitable for good quality biodiesel. ${ }^{63}$ Unsaturated fatty acids in PCC7942, particularly C18:1, have been reported to increase under iron starvation. ${ }^{64}$ In this way, iron starvation could serve as

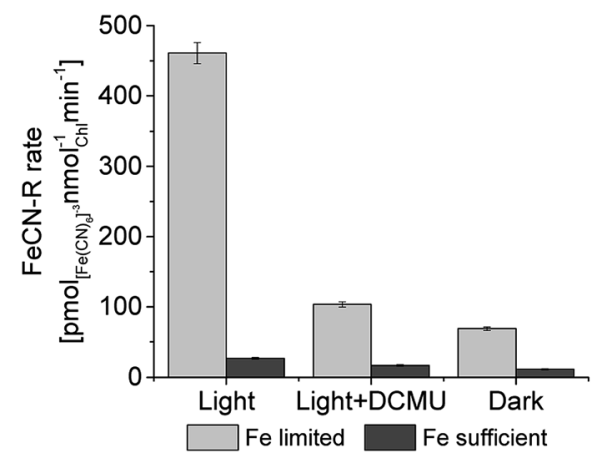

Fig. 6 Ferricyanide reduction rates of iron limited cultures and iron sufficient cultures of PCC7942, in light, in light plus the photosynthesis inhibitor DCMU and in darkness (assayed at $\mathrm{pH}$ 7.0). FeCN-R rate $n=3$ \pm 1SE. a strategy for an integrated system with biomass growth for biodiesel production coupled to photo-bioelectricity generation.

\section{Comparative analysis of PCC7942 exoelectrogenic capacity}

Light driven FeCN-R rates of $460 \mathrm{pmol}_{\left(\left[\mathrm{Fe}(\mathrm{CN})_{6}\right]^{3-}\right)} \mathrm{nmol}_{\mathrm{Chl}}{ }^{-1} \mathrm{~min}^{-1}$ is unprecedented for cyanobacteria (Table 1). In Synechocystis sp. 6803 deletion of all respiratory cytochrome oxidases resulted in

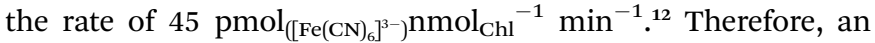
intracellular redox imbalance due to blockage of terminal oxidases due to the lack of iron is unlikely the cause of the high rates obtained in iron limited PCC7942, which achieved rates 10 times higher than those of Synechocystis sp. 6803 respiratory terminal oxidase (rto) mutants. In high salt, PCC6803 achieved a reduction rate of $50 \mathrm{pmol}_{\left(\left[\mathrm{Fe}(\mathrm{CN})_{6}\right]^{3-}\right)} \mathrm{nmol}_{\mathrm{Chl}}{ }^{-1} \mathrm{~min}^{-1}$, while rto mutants in high salt achieved the relatively high rate of 135 $\mathrm{pmol}_{\left(\left[\mathrm{Fe}(\mathrm{CN})_{6}\right]^{3-}\right)} \mathrm{nmol}_{\mathrm{Chl}}{ }^{-1} \mathrm{~min}^{-1}{ }^{65}$ still less than a third of that achieved by the synergistic effect of neutral $\mathrm{pH}$ and iron limitation in wild-type PCC7942. FeCN-R rates obtained for iron sufficient PCC7942 at neutral pH, especially with young cultures, are similar to those achieved by Synechocystis sp. PCC6803 rto mutant (low salt) and to the rates induced by high salt in wildtype Synechocystis sp. PCC6803. The effect of neutral pH alone resulted in rates as high as those achieved by these other strategies.

Synechocystis sp. PCC6803 rto mutants showed similar rates in the light and in the dark, meaning that in the dark and in high salt rto mutants reached rates around $130 \operatorname{pmol}_{\left(\left[\mathrm{Fe}(\mathrm{CN})_{6}\right]^{3-}\right)^{-}}$ $\mathrm{nmol}_{\mathrm{Chl}}{ }^{-1} \mathrm{~min}^{-1}{ }^{65}$ In the dark, the FeCN-R rates measured for iron limited PCC7942 were high as well, reaching 70 $\mathrm{pmol}_{\left(\left[\mathrm{Fe}(\mathrm{CN})_{6}{ }^{3-}\right)\right.} \mathrm{nmol}_{\mathrm{Chl}^{-1}} \mathrm{~min}^{-1}$, also a significant improvement, but it is in the light where the major contribution was made. The latter shows how the strategies are based on different and parallel approaches. While rto mutants inhibit respiration, diverting reducing power mainly from respiration, iron limitation resulted in diversion of reducing power directly from photosynthesis.

The outer membrane cytochrome OmcS has been proven to reduce Fe(III) oxides, ${ }^{66}$ and when introduced into PCC7942, it demonstrated to also reduce ferricyanide. In the PCC7942 strain harbouring the OmcS, FeCN-R rates were improved to 35

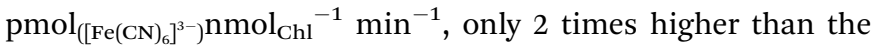


Table 1 Strategies to induce higher ferricyanide reductase activity in photosynthetic microorganisms. Rates are presented in two equivalent units in order to compare with reported values and within microalgae and cyanobacteria. All studies were conducted at $30{ }^{\circ} \mathrm{C}$ except $\mathrm{C}$. reinhardtii which was conducted at $20^{\circ} \mathrm{C}$. The control for FeCN-R rates in PCC7942 is iron sufficient cultures at alkaline $\mathrm{pH}$

Ferricyanide reduction rate in the light

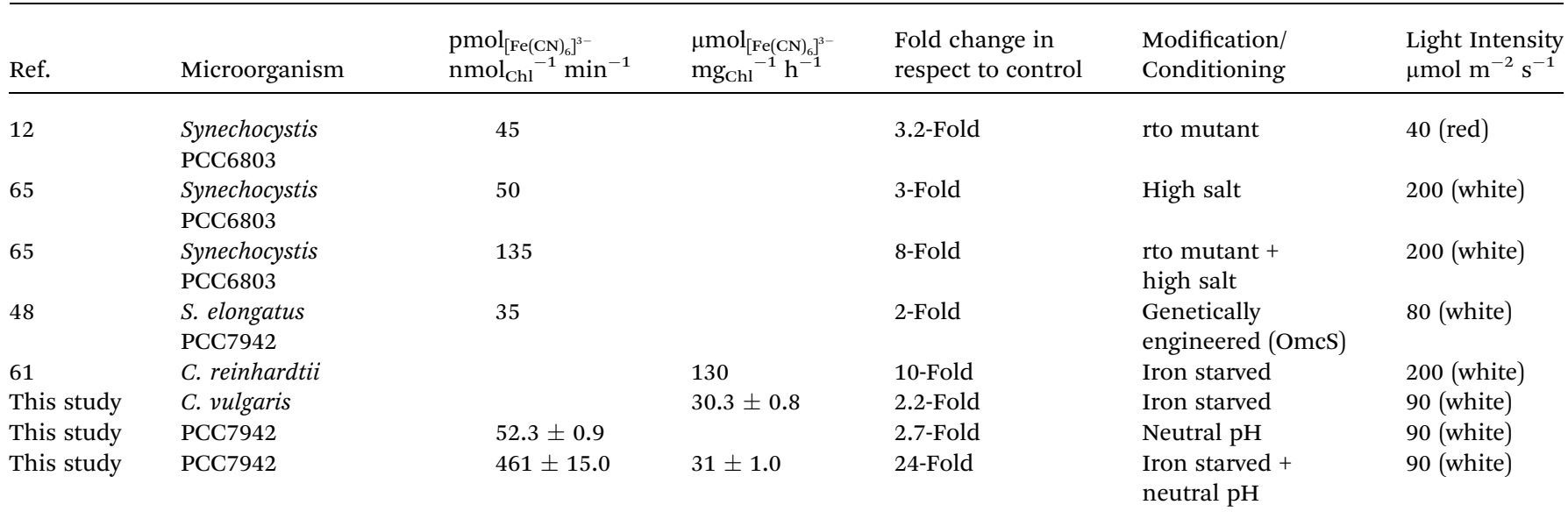

rates for the wild-type. ${ }^{48}$ The latter indicates that in addition to the introduced outer membrane c-type cytochrome, there is a constitutional mechanism redirecting electrons to the outer membrane, which was available for the c-cytochrome to react. Under normal conditions, this activity is low, but under stress conditions, such as neutral $\mathrm{pH}$ and especially in iron starvation, this reductive mechanism is enhanced. Iron limited PCC7942 (wild-type) achieved rates over 13 times higher than PCC7942 harbouring OmcS, therefore the sole incorporation of cytochromes in the outer membrane is not sufficient to achieve rates like those induced in iron limitation, which triggers an intracellular redirection of electrons to the extracellular.

In relation to FeCN-R activity in microalgae, which are known to possess ferric reductases in the cell surface, higher values have been reported for $C$. reinhardtii. ${ }^{61}$ Nonetheless, light intensities and spectrum (colour) differ in the different studies, implying that rates in the light might be not entirely comparable. For instance, $C$. reinhardtii was tested under a light intensity of 200 $\mu \mathrm{mol} \mathrm{m}{ }^{-2} \mathrm{~s}^{-1}$ (white light), which was more than twice this study. In order to compare to microalgae in the same conditions, as part of this investigation the microalgae $C$. vulgaris grown in the same conditions than PCC7942 was evaluated (ESI Fig. $2 \dagger$ ), finding that the FeCN-R activity of iron limited C. vulgaris (ESI Fig. $3 \dagger$ ) was similar to that of iron limited PCC7942.

Screening for other cyanobacteria strains with irondeficiency-induced exoelectrogenesis is a way forward. A thermophilic strain of the species Synechococcus elongatus was reported to increase ferric-EDTA reduction rates in iron starvation (3 days). These rates were very low if compared to the FeCN-R rates, but still there was a 10 -fold increment, ${ }^{36}$ implying the trait could be common among the species.

\section{Mediatorless electrochemical detection of extracellular activity in PCC7942}

While using ferricyanide as a redox shuttle facilitates the transfer of electrons from the cyanobacterial cell to the anode, mediatorless systems are more sustainable. Ferricyanide is a small molecule and it can cross porin channels in the outer membrane, accessing to the periplasm and plasma membrane. However, ferricyanide could also be reacting at the outer membrane. The latter is exemplified by the increase in FeCN-R rates by PCC7942 with the sole incorporation of the ccytochrome OmcS in the outer membrane. ${ }^{48}$

Voltammetry studies of cyanobacteria on graphite rods functionalised with immobilised redox mediators (osmium and azine redox polymers) showed slightly higher peak currents in the light than in the dark, but the signal still corresponds to that of the mediator. ${ }^{67}$ Despite of mediatorless photo-bioelectricity generation being demonstrated for a variety of photosynthetic microorganisms, an electrochemical characterisation of the redox complexes involved in their exoelectrogenic capacity has been elusive.

To investigate and characterise the mediatorless redox interaction of PCC7942 on an electrode, biofilms were prepared by leaving cells to grow over ITO-coated glass at the bottom of an electrochemical device (as shown in Fig. 2). SEM images showed the effect of repeated washing steps on the thickness of the biofilm (Fig. 7(A)). Fig. 7(B) pictures the working electrodes with thick (left) and thin (right) biofilms, as observed, it is clear to see at the naked eye that by washing off the loosely attached cells the biomass density was reduced.

Cyclic voltammetry of biofilms of PCC7942, in absence of any mediator, showed electrochemical activity in the dark and in the light (Fig. 8, 9 and 10). As expected, biofilms under illumination were more reactive (Fig. 10) and thinner biofilms generated smaller currents (Fig. 9). In the dark, only thick biofilms showed significant currents.

In the dark (Fig. 8), the potential detected at zero current (OCP vs. $\mathrm{Ag} / \mathrm{AgCl}$ ) was around $0.18 \mathrm{~V}$ vs. $\mathrm{Ag} / \mathrm{AgCl}$ for both iron limited and iron sufficient biofilms. No plateau current was observed, nor a clear anodic peak within the potential window studied. However, an increase in the current signal is observed, 
(A)

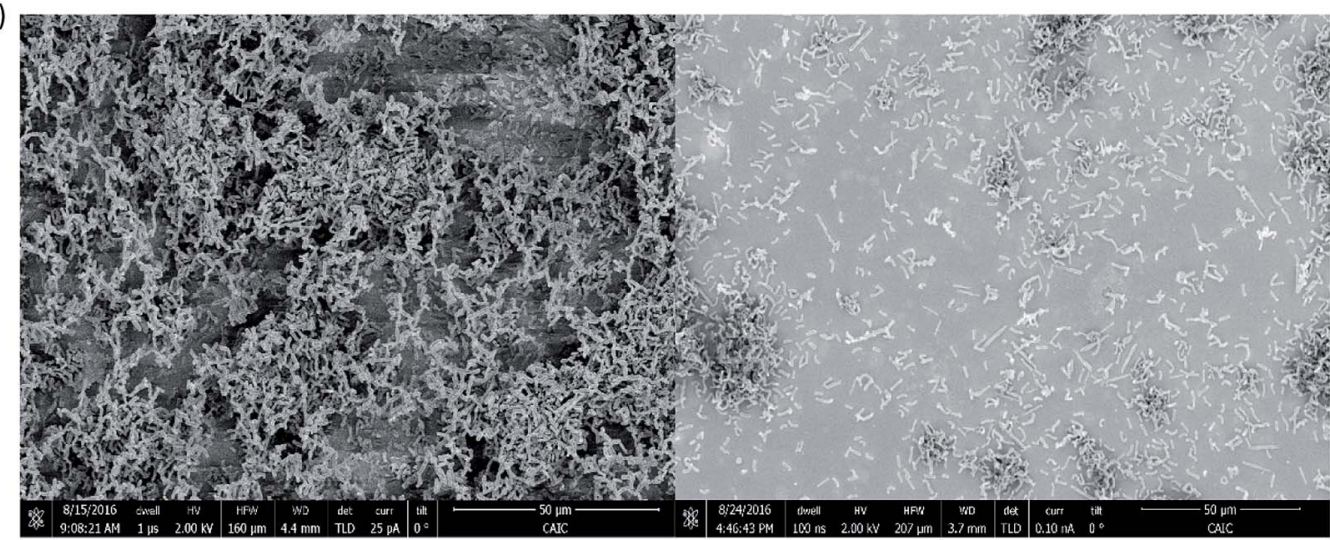

(B)

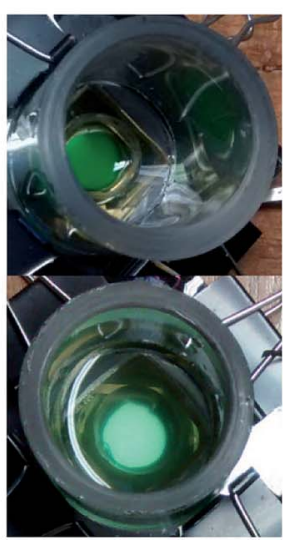

Fig. 7 (A) SEM images of PCC7942 biofilms on ITO-coated glass, biofilm with gentle washing (left) and biofilm with repeated washing (right). (B) Photography of working electrodes with thick biofilm (up) and thin biofilm (bottom) by washing off loosely attached cells.

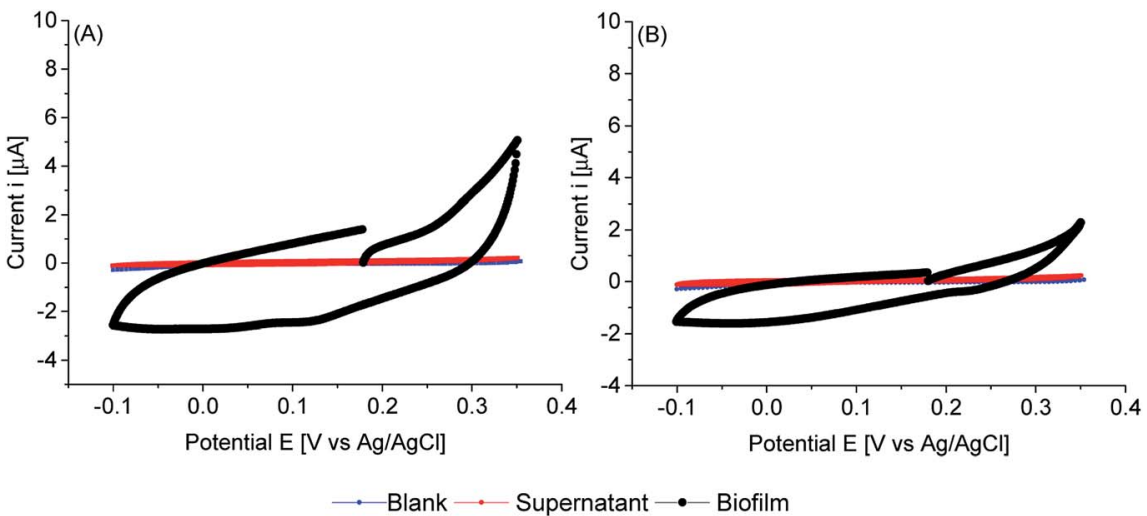

Fig. 8 Cyclic voltammogram obtained in darkness from PCC7942 thick biofilms on ITO-coated glass, without artificial redox mediator in (A) iron limitation and (B) iron sufficiency. Measurements conducted in darkness at $30{ }^{\circ} \mathrm{C}(\mathrm{pH} 7.30-7.40)$. Scan rate $1 \mathrm{mV} \mathrm{s}^{-1}$. Initial potential [OCP -50 $\mathrm{mV}]$ vs. Ag/AgCl. Devices were inoculated with the same amount of biomass; however, the density of attached cells might differ.

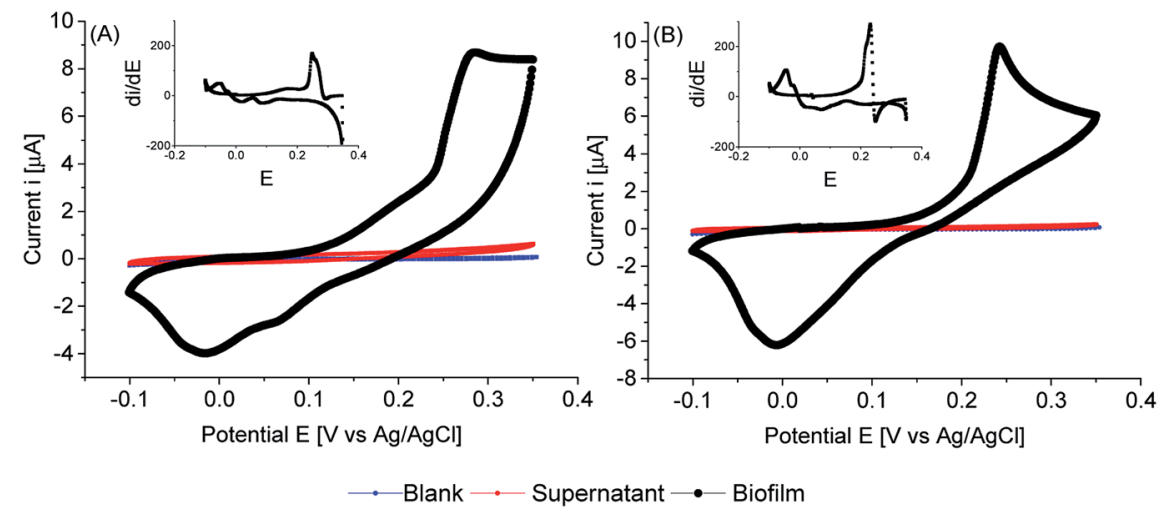

Fig. 9 Cyclic voltammogram obtained under illumination from thick PCC7942 biofilms on ITO-coated glass, without artificial redox mediator in (A) iron limitation and (B) iron sufficiency. Measurements conducted in light at $30{ }^{\circ} \mathrm{C}(\mathrm{pH} 7.30-7.40)$. Scan rate $1 \mathrm{mV} \mathrm{s}^{-1}$. Initial potential [OCP $50 \mathrm{mV}$ vs. Ag/AgCl. Devices were inoculated with the same amount of biomass; however, the density of attached cells might differ. Insets: first derivative di/dE current change over potential.

particularly in iron limited biofilms. In the back sweep, cathodic currents appeared at high positive potentials. All indicating that biofilms adapted in the dark were in a pseudo oxidised state, hence accepting electrons. However, in the back sweep biofilms were reduced and as potentials were swept forward, anodic currents appeared at around $0.0 \mathrm{~V} \mathrm{vs.} \mathrm{Ag} / \mathrm{AgCl}$.

This potential, $0.0 \mathrm{~V} v s$. $\mathrm{Ag} / \mathrm{AgCl}$, coincides with the potential at zero current when biofilms were illuminated (Fig. 9). 


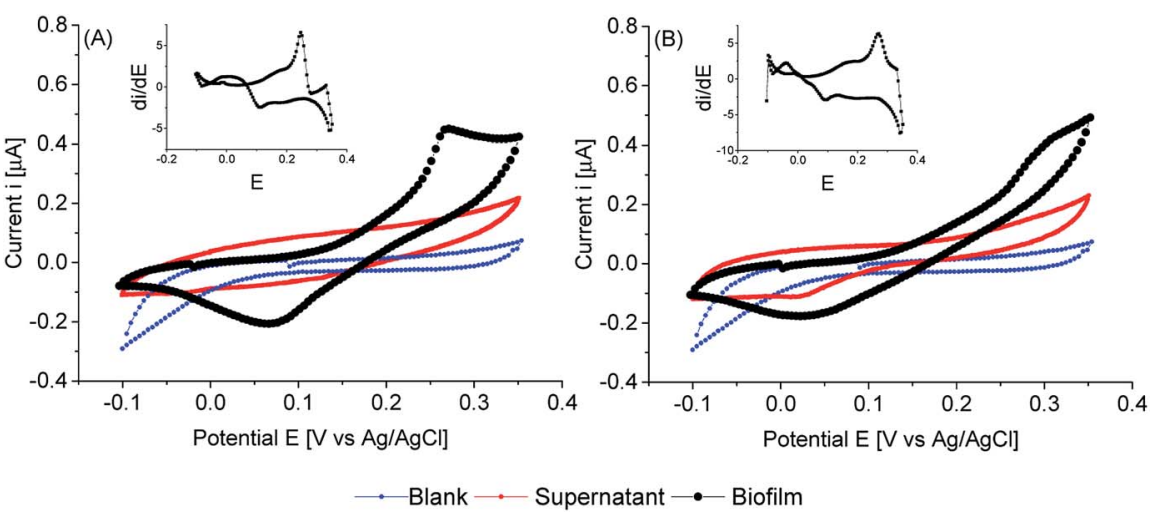

Fig. 10 Cyclic voltammogram obtained under illumination from thin PCC7942 biofilms on ITO-coated glass, without artificial redox mediator in (A) iron limitation and (B) iron sufficiency. Measurements conducted in light at $30^{\circ} \mathrm{C}(\mathrm{pH} 7.30-7.40)$. Scan rate $1 \mathrm{mV} \mathrm{s} \mathrm{s}^{-1}$. Initial potential [OCP $50 \mathrm{mV}] \mathrm{vs}$. Ag/AgCl. Devices were inoculated with the same amount of biomass; however, the density of attached cells might differ. Insets: first derivative di/dE current change over potential.

However, a large activation polarisation loss was seen, especially in the iron sufficient biofilm, implying sluggish kinetics. It can be observed that in the region of $0.1-0.2 \mathrm{~V} v s$. $\mathrm{Ag} / \mathrm{AgCl}$, iron limited biofilms showed an increased in currents while iron sufficient biofilms currents remained lower. However, a large increase in currents developed at higher potentials for both iron limited and iron sufficient biofilms, with a starting potential around $0.20 \mathrm{~V}$ vs. $\mathrm{Ag} / \mathrm{AgCl}$ and a marked midpoint potential around $0.22 \mathrm{~V} v s$. $\mathrm{Ag} / \mathrm{AgCl}$, which in turn coincides with the potential region that biofilms adapted in the dark showed the initial potential value. The midpoint potential is better visualised in the first derivative of the current (insets Fig. 9). It appears that PCC7942 biofilms may present two distinctive electroactive regions. Nonetheless, higher reactivity happens in the potential region $0.20-0.25 \mathrm{~V} v \mathrm{~s}$. Ag/AgCl.

Remarkably, biofilms grown from iron limited cultures showed in general larger anodic currents in both dark and light conditions, demonstrating that the enhanced exoelectrogenic activity in iron limited PCC7942 is, at least partially, independent of a soluble artificial redox mediator. Interestingly, illuminated iron limited biofilms were able to sustain currents at higher potentials in a similar fashion to that seen in exoelectrogenic bacteria (sigmoidal-like curve), meaning saturating levels of electron donor and limiting current due to the catalytic rates of enzymatic activity $\left(V_{\max }\right)^{68,69}$ Differently, currents in iron sufficient biofilms drastically dropped, showing a pronounced peak, in a diffusion-limiting fashion. ${ }^{68}$ In the case of biofilms, the availability of electron donor at the electrode is the result of intracellular processes, which for bacteria also depend on the diffusion of an organic substrate, but in the case of photosynthetic biofilms, the availability of electron donor is not readily manipulated and it depends on the conditions of photosynthetic growth, mainly light intensity, but an external substrate diffusion step is not present, eliminating some complexity in the analysis. Therefore, the current drop is likely due to an exhausted biofilm, meaning that electrons are not being replenished at a fast-enough rate and indicating there might be a metabolic limitation. This metabolic limitation can be exacerbated in thick biofilms, where the deeper layers are shaded, affecting photosynthetic rates.
When thinner biofilms of PCC7942 were tested, the magnitude of the currents was lower. The resulting voltammograms are shown in Fig. 10. Compared with the thicker biofilms (Fig. 9), there is a substantial difference in current densities, demonstrating that the thicker biofilms provided a larger electron pool and demonstrating that relay of electrons in cell to cell interaction occurred. In Fig. 11 is observed that biofilms of PCC7942 form an intricate extracellular matrix and evident pilus structures interconnecting the cells are seen. The capacity of extracellular electron transfer in bacteria has been associated with electrical conductive nanowires (pili), which have been characterised in S. oneidensis and in G. sulfurreducens. ${ }^{20,70}$ Cell to cell interaction allegedly allows electron relay through the biofilm.

In comparison with thick biofilms, activation polarisation losses were lower and further analysis of the first derivative plot (insets Fig. 10), confirmed that there was higher activity in the region of the first peak in respect to the second peak. Furthermore, the first electroactive region is also observed in the iron sufficient biofilm. It can be concluded that thick biofilms conferred higher resistivity, possibly related to cells reacting in distant layers.

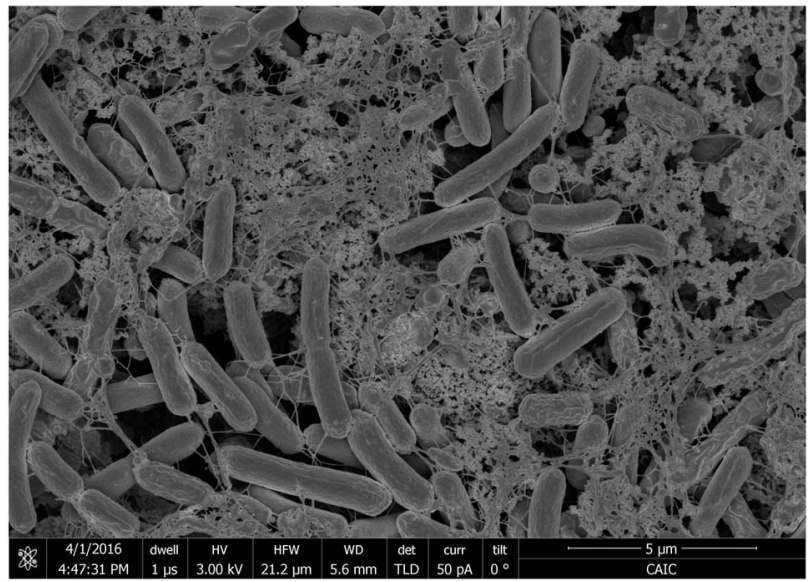

Fig. 11 SEM image of PCC7942 biofilm showing pili structures allowing cell to cell interactions. 
Iron limited thin biofilms showed a similar waveform to thick biofilms, showing a tendency to plateau currents. Differently, in the iron sufficient biofilm, a pronounced peak was not seen, but there was the corresponding mid-point potential peak (visible on the inset). Therefore, it appears that light penetration had an impact on electron availability. Nonetheless, there was an obvious higher resistivity on iron sufficiency (poor charge transfer) compared with iron limitation. The latter reached $V_{\max }$, while the iron sufficient biofilm required higher overpotentials to drive larger currents.

To the best of our knowledge this is the first time that a cyanobacterial biofilm shows a clear electrochemical active region in a mediatorless system, showing an electrochemical signature of complexes at the outer membrane level. Using electrochemical techniques like voltammetry will facilitate the search for adequate microorganisms and electrode materials.

It is remarkable that iron starvation generated an intracellular electron pool available extracellularly in a saturated-like manner, indicating that the pathway involved was very active in redirecting electrons to the extracellular environment. Furthermore, it was proven that the exoelectrogenic mechanism behind iron limitation might be involved in a pathway acting at outer membrane level. The electrochemical active regions of iron sufficient biofilms were the same, therefore the mediatorless detection of electricity in PCC7942 (normal conditions) is possibly due to the basal activity of the alleged reductive iron acquisition system. All in all, PCC7942 can be used in the more sustainable mediatorless platforms for photo-bioelectricity generation and iron limited growth is a tool which can be exploited to increase current densities.

\section{Conclusions}

The present investigation has demonstrated that the exoelectrogenesis capacity of PCC7942 can be conditioned by means of environmental factors. Remarkably, PCC7942 grown in iron limited conditions acquires significantly enhanced exoelectrogenic capacity, which is higher at neutral $\mathrm{pH}$, comparable to that of ferric reductases in algae. The use of ferricyanide as a mediator facilitates the transfer of electrons from the cell envelope, however it was shown that PCC7942 mediatorless interaction with an electrode is also enhanced in the iron limited condition.

Iron limited PCC7942 exoelectrogenesis confers good properties for the operation of biophotovoltaics, in terms of a higher electron availability and in terms of the $\mathrm{pH}$ of the system, which will improve both the anodic and the cathodic potential. Exoelectrogenic capacity in PCC7942 is mainly photosynthesis driven with redirection of electrons directly from the light reactions. Nonetheless, the increased exoelectrogenic activity detected in the dark is also a significant improvement for a light-driven platform. Bioelectricity generation conditioned by iron limitation demonstrates a way to externally manipulate the metabolism of cyanobacteria to increase current densities, providing a starting point to engineer the system for constitutive high exoelectrogenic capacity. We envision the development of a technology using the exoelectrogenic capacity of cyanobacteria to recover energy from photo-bioreactors for generation of bio-products, for instance biodiesel, improving the sustainability of the process.

\section{Conflicts of interest}

There are no conflicts to declare.

\section{Acknowledgements}

The authors are grateful for funding support provided by Becas Chile - Conicyt together with Cambridge Trust to A. C. Gonzalez-Aravena, for funding by CARES CAM.CREATE C4T and by the British Council Newton Fund Prize to A. C. Fisher, and by M4080306 NTU (Singapore) to B. Norling. We thank Dr Tiago Selao for helpful discussions, we also thank Dr Kamal Elouarzaki and Dr Dongda Zhang for their valuable comments.

\section{References}

1 A. Elmekawy, H. M. Hegab, K. Vanbroekhoven and D. Pant, Renewable Sustainable Energy Rev., 2014, 39, 617-627.

2 A. P. Borole, G. Reguera, B. Ringeisen, Z.-W. Wang, Y. Feng and B. H. Kim, Energy Environ. Sci., 2011, 4, 4813.

3 O. Bretschger, A. Obraztsova, C. a Sturm, S. Chang, Y. a Gorby, S. B. Reed, D. E. Culley, C. L. Reardon, S. Barua, M. F. Romine, J. Zhou, A. S. Beliaev, R. Bouhenni, D. Saffarini, F. Mansfeld, B.-H. Kim, J. K. Fredrickson and K. H. Nealson, Appl. Environ. Microbiol., 2007, 73, 7003-7012. 4 P. Bombelli, R. W. Bradley, A. M. Scott, A. J. Philips, A. J. McCormick, S. M. Cruz, A. Anderson, K. Yunus, D. S. Bendall, P. J. Cameron, J. M. Davies, A. G. Smith, C. J. Howe and A. C. Fisher, Energy Environ. Sci., 2011, 4, 4690.

5 R. I. Pinhassi, D. Kallmann, G. Saper, H. Dotan, A. Linkov, A. Kay, V. Liveanu, G. Schuster, N. Adir and A. Rothschild, Nat. Commun., 2016, 7, 12552.

6 H. A. Dewi, F. Meng, B. Sana, C. Guo, B. Norling, X. Chen and S. Lim, RSC Adv., 2014, 4, 48815.

7 A. J. McCormick, P. Bombelli, A. M. Scott, A. J. Philips, A. G. Smith, A. C. Fisher and C. J. Howe, Energy Environ. Sci., 2011, 4, 4699.

8 C.-C. Lin, C.-H. Wei, C.-I. Chen, C.-J. Shieh and Y.-C. Liu, Bioresour. Technol., 2013, 135, 640-643.

9 B. De Caprariis, P. De Filippis, A. Di Battista, L. Di and M. Scarsella, Chem. Eng. Trans., 2014, 38, 523-528.

10 A. J. McCormick, B. Paolo, R. W. Bradley, R. Thorne, T. Wenzel and C. J. Howe, Energy Environ. Sci., 2015, 8, 1092-1109.

11 N. Quintana, F. Van der Kooy, M. D. Van de Rhee, G. P. Voshol and R. Verpoorte, Appl. Microbiol. Biotechnol., 2011, 91, 471-490.

12 R. W. Bradley, P. Bombelli, D. J. Lea-Smith and C. J. Howe, Phys. Chem. Chem. Phys., 2013, 15, 13611.

13 R. W. Bradley, P. Bombelli, S. J. L. Rowden and C. J. Howe, Biochem. Soc. Trans., 2012, 40, 1302-1307.

14 D. J. Lea-Smith, P. Bombelli, R. Vasudevan and C. J. Howe, Biochim. Biophys. Acta, Bioenerg., 2016, 1857, 247-255. 
15 R. A. Bouhenni, G. J. Vora, J. C. Biffinger, S. Shirodkar, K. Brockman, R. Ray, P. Wu, B. J. Johnson, E. M. Biddle, M. J. Marshall, L. A. Fitzgerald, B. J. Little, J. K. Fredrickson, A. S. Beliaev, B. R. Ringeisen and D. A. Saffarini, Electroanalysis, 2010, 22, 856-864.

16 Y. Xiong, L. Shi, B. Chen, M. U. Mayer, B. H. Lower, Y. Londer, S. Bose, M. F. Hochella, J. K. Fredrickson and T. C. Squier, J. Am. Chem. Soc., 2006, 128, 13978-13979.

17 D. R. Lovley, Annu. Rev. Microbiol., 2012, 66, 391-409.

18 H. von Canstein, J. Ogawa, S. Shimizu and J. R. Lloyd, Appl. Environ. Microbiol., 2008, 74, 615-623.

19 E. Marsili, D. B. Baron, I. D. Shikhare, D. Coursolle, J. a Gralnick and D. R. Bond, Proc. Natl. Acad. Sci. U. S. A., 2008, 105, 3968-3973.

20 T. J. Beveridge, I. S. Chang, B. H. Kim, S. Kim, D. E. Culley, S. B. Reed, F. Margaret, D. A. Saffarini, E. A. Hill, L. Shi, A. Dwayne, Y. A. Gorby, S. Yanina, J. S. Mclean, K. M. Rosso, D. Moyles, A. Dohnalkova, K. S. Kim, M. F. Romine, D. A. Elias and D. W. Kennedy, Proc. Natl. Acad. Sci., 2009, 106, 9535.

21 M. Y. El-Naggar, G. Wanger, K. M. Leung, T. D. Yuzvinsky, G. Southam, J. Yang, W. M. Lau, K. H. Nealson and Y. a Gorby, Proc. Natl. Acad. Sci. U. S. A., 2010, 107, 1812718131.

22 J. C. B. Fitzgerald, A. Lisa, E. R. Petersen, R. I. Ray, B. J. Little, C. J. Cooper, E. C. Howard and B. R. Ringeisen, Process Biochem., 2012, 47, 170-174.

23 D. Baron, E. LaBelle, D. Coursolle, J. a Gralnick and D. R. Bond, J. Biol. Chem., 2009, 284, 28865-28873.

24 E. Marsili, J. B. Rollefson, D. B. Baron, R. M. Hozalski and D. R. Bond, Appl. Environ. Microbiol., 2008, 74, 7329-7337.

25 A. Anderson, A. Laohavisit, I. K. Blaby, P. Bombelli, C. J. Howe, S. S. Merchant, J. M. Davies and A. G. Smith, Plant Biotechnol. J., 2016, 14, 22-28.

26 X. Zhang, K. H. Krause, I. Xenarios, T. Soldati and B. Boeckmann, PLoS One, 2013, 8, e58126.

27 J. A. Lynnes, T. L. M. Derzaph and H. G. Weger, Planta, 1998, 204, 360-365.

28 M. Zhou, H. He, T. Jin and H. Wang, J. Power Sources, 2012, 214, 216-219.

29 A. Laohavisit, A. Anderson, P. Bombelli, M. Jacobs, C. J. Howe, J. M. Davies and A. G. Smith, Algal Res., 2015, 12, 91-98.

30 R. J. Thorne, K. Schneider, H. Hu and P. J. Cameron, Bioelectrochemistry, 2015, 105, 103-109.

31 S. W. Wilhelm and C. G. Trick, Limnol. Oceanogr., 1994, 39, 1979-1984.

32 S. W. Wilhelm, D. P. Maxwell and C. G. Trick, Limnol. Oceanogr., 1996, 41, 89-97.

33 R. Raghuvanshi, S. Singh and P. S. Bisen, Indian J. Exp. Biol., 2007, 45, 563-567.

34 C. Kranzler, H. Lis, Y. Shaked and N. Keren, Environ. Microbiol., 2011, 13, 2990-2999.

35 H. Lis, C. Kranzler, N. Keren and Y. Shaked, Life, 2015, 5, 841-860.

36 B. K. Semin, L. N. Davletshina, A. a Novakova, T. Y. Kiseleva, V. Y. Lanchinskaya, A. Y. Aleksandrov, N. Seifulina,
I. I. Ivanov, M. Seibert and A. B. Rubin, Plant Physiol., 2003, 131, 1756-1764.

37 J. J. Lamb, R. E. Hill, J. J. Eaton-Rye and M. F. HohmannMarriott, PLoS One, 2014, 9, e105761.

38 C. Kranzler, H. Lis, O. M. Finkel, G. Schmetterer, Y. Shaked and N. Keren, ISME J., 2014, 8, 409-417.

39 H.-B. Jiang, W.-J. Lou, W.-T. Ke, W.-Y. Song, N. M. Price and B.-S. Qiu, ISME J., 2015, 9, 297-309.

40 N. Yousef, E. K. Pistorius and K.-P. Michel, Arch. Microbiol., 2003, 180, 471-483.

41 K.-P. Michel, E. K. Pistorius and S. S. Golden, J. Bacteriol., 2001, 183, 5015-5024.

42 E. J. Boekema, A. Hifney, a E. Yakushevska, M. Piotrowski, W. Keegstra, S. Berry, K. P. Michel, E. K. Pistorius and J. Kruip, Nature, 2001, 412, 745-748.

43 K. Michel and E. Pistorius, Physiol. Plant., 2004, 120, 36-50. 44 a. G. Ivanov, Y.-I. Park, E. Miskiewicz, J. a. Raven, N. P. a. Huner and G. Öquist, FEBS Lett., 2000, 485, 173-177.

45 D. A. Bryant, The Molecular Biology of Cyanobacteria Volume I and II, Springer-Science+Business Media, B.V., 1994.

46 C. G. Trick and A. Kerry, Curr. Microbiol., 1992, 24, 241-245. 47 A. Nodop, D. Pietsch, R. Höcker, A. Becker, E. K. Pistorius, K. Forchhammer and K.-P. Michel, Plant Physiol., 2008, 147, 747-763.

48 N. Sekar, R. Jain, Y. Yan and R. P. Ramasamy, Biotechnol. Bioeng., 2016, 113, 675-679.

49 R. J. Ritchie, Photosynth. Res., 2006, 89, 27-41.

50 M. S. Davey, D. J. Suggett, R. J. Geider and A. R. Taylor, 2003, 1144, 1132-1144.

51 Q. Hu, P. Westerhoff, W. Vermaas, Q. Hu and P. Westerhoff, 2000, 66, 133-139.

52 D. Kuan, S. Duff, D. Posarac and X. Bi, Can. J. Chem. Eng., 2015, 93, 640-647.

53 A. Marchler-Bauer and S. H. Bryant, Nucleic Acids Res., 2004, 32, 327-331.

54 W. Schmidt, New Phytol., 1999, 141, 1-26.

55 J. Rodríguez-Celma and W. Schmidt, Plant Signaling Behav., 2013, 8, e26116.

56 W. Schmidt, W. Michalke and A. Schikora, Plant, Cell Environ., 2003, 26, 361-370.

57 S. Santi and W. Schmidt, New Phytol., 2009, 183, 1072-1084. 58 U. Eckhardt and T. J. Buckhout, J. Exp. Bot., 1998, 49, 12191226.

59 J. D. Hem, Geol. Soc. Am. Bull., 1972, 83, 443-450.

60 E. Alcantara, M. D. De Guardia, F. J. Romera, M. D. Delaguardia, E. Alcántara and M. D. de la Guardia, Plant Physiol., 1991, 96, 1034-1037.

61 X. Xue, C. M. Collins, H. G. Weger and A. Fecn-r, J. Phycol., 1998, 34, 939-944.

62 J. A. Raven, M. C. W. Evans and R. E. Korb, Photosynth. Res., 1999, 60, 111-149.

63 C. S. P. Silva, M. E. Silva-Stenico, M. F. Fiore, H. F. De Castro and P. C. M. Da Ros, Algal Res., 2014, 3, 1-7.

64 A. G. Ivanov, M. Krol, E. Selstam, P. V. Sane, D. Sveshnikov, Y. Il Park, G. Öquist and N. P. A. Huner, Biochim. Biophys. Acta, 2007, 1767, 807-813. 
65 A. J. McCormick, P. Bombelli, D. J. Lea-Smith, R. W. Bradley, A. M. Scott, A. C. Fisher, A. G. Smith and C. J. Howe, Energy Environ. Sci., 2013, 6, 2682.

66 M. Aklujkar, M. V. Coppi, C. Leang, B. C. Kim, M. A. Chavan, L. A. Perpetua, L. Giloteaux, A. Liu and D. E. Holmes, Microbiol., 2013, 159, 515-535.

67 K. Hasan, H. Bekir Yildiz, E. Sperling, P. O Conghaile, M. a Packer, D. Leech, C. Hägerhäll and L. Gorton, Phys. Chem. Chem. Phys., 2014, 16, 24676-24680.
68 E. Labelle and D. R. Bond, in Bio-electrochemical systems: from extracellular electron transfer to biotechnological application, ed. D. I. P. Lens, Integrated Environmental Technology Series, The Netherlands, 2009, pp. 1-9.

69 C. I. Torres, A. K. Marcus, H.-S. Lee, P. Parameswaran, R. Krajmalnik-Brown and B. E. Rittmann, FEMS Microbiol. Rev., 2010, 34, 3-17.

70 C. Leang, X. Qian, T. Mester and D. R. Lovley, Appl. Environ. Microbiol., 2010, 76, 4080-4084. 